\title{
An Optical CDMA Random Access Protocol for Multi-rate Optical Networks Adopting Multi-coding Techniques
}

\author{
Amira M. Shata|*, Shimaa A. Mohamed |*, Ahmed Abdel Nabi*, and Hossam M. H. Shalaby||** \\ ||Department of Electrical Engineering, Faculty of Engineering, Alexandria, Egypt. \\ *Informatics Research Institute, City of Scientific Research and Technological Applications, Alexandria, Egypt. \\ **Dept. of Electron. and Commun. Eng., Egypt-Japan University of Science and Technology (E-JUST), Alexandria, Egypt \\ amirashata88@gmail.com, shimaaabdellatif.2011@gmail.com, iplanetfit@yahoo.com, shalaby@ieee.org
}

\begin{abstract}
A link layer protocol that supports multi-rate on OCDMA network is proposed based on multi-coding techniques to improve the performance of the network. Our objective is to allow users of different data rates to access the network simultaneously without affecting or reducing the networks performance. Signature sequences are achieved using OCFHC/OOC 2D codes. System throughput and average packet delay are derived for two receiver models, namely, the correlation and chip-level receivers. In our analysis, we only take the effect of multiple access interference (MAI) into account and neglect the effect of receiver noise, as it is relatively minor. The network users are classified into two classes with two different transmission rates. Furthermore, the number of classes is generalized and we are able to show that the overall performance of the network is even better.
\end{abstract}

Keywords - Multi-coding, multi-rate, one coincidence frequency hop code/optical orthogonal code (2D OCFHC/OOC), optical code-division multiple-access (OCDMA), packet delay, throughput.

\section{INTRODUCTION}

Optical code division multiple access (OCDMA) has been regarded as one of the most promising technologies for the next generation of optical access networks [1]. In an incoherent OCDMA system, each user is allocated a unique signature sequence from a family of $0 / 1$ sequences referred to as the optical orthogonal codes (OOCs) that satisfy certain correlation properties [2].

Owing to the increasing interest in the multi-media applications, networks supporting heterogeneous traffic, e.g., high and low-speed data, text, image, audio, and video with varieties of quality of service and traffic requirements, have become indispensable. Multi-coding techniques have been introduced in [3] for optical code-division multiple-access (OCDMA) systems. In this technique, traffic streams with different transmission rates are integrated into a unified architecture. Users of high rate are assigned a number of codes based on the relation between the rate they are requesting and the basic rate of the system. To overcome the shortcoming of one-dimensional optical orthogonal code (1D OOC), we adopt two-dimensional optical orthogonal code called (2D OCFHC/OOC), which extends the cardinality. 2D OCFHC/OOC, unlike other 2D optical codes, supports good cardinality and has very good correlation properties [4].

In this paper, we extend the random-access protocol for the OCDMA network proposed in [5] for single-rate users to be suitable for multi-rate users.

The remainder of this paper is organized as follows: Section II discusses a basic description of the system architecture. The system model and the theoretical analysis are presented in section III. The numerical results are presented in Section IV. Finally, the conclusion is given in Section V.

\section{SYSTEM ARCHITECTURE}

The basic architecture of the proposed optical CDMA network is composed of a set of $N$ nodes or users, an optical star network, and a set of one coincidence frequency hop code/Optical orthogonal code (OCFHC/OOC), which is represented by a $p^{k} \times N_{\text {ooc }}$ matrix consisting of $0 \mathrm{~s}$ and $1 \mathrm{~s}$ entries. Here, $p$ is a prime number, $k$ is a positive integer, $p^{k}$ is the number of wavelengths of the $2 \mathrm{D}$ code, and $N_{o o c}$ is the OOC code length. The cardinality $C$ of an OCFHC/OOC is given by [4]

$$
C=p^{k}\left(p^{k}-1\right) \frac{N_{o o c}-1}{w(w-1)}
$$

where $w$ is the OOC code weight. A one-coincidence frequency hop code (OCFHC)/OOC employs OCFHC and $\mathrm{OOC}$ as wavelength hopping and time-spreading patterns, respectively. OCFHC/OOC has very good correlation properties and is independent of the number of wavelength and code length in time domain [4].

\section{III.SYSTEM MODEL AND ANALYSIS}

We consider an optical CDMA network of $N$ users. Each user belongs to one of two available classes: class I and class II. A class I user sends $m \in\{1,2,3, \ldots\}$ packets/slot, whereas a class II user sends 1 packet/slot. That is the transmission rate 
of a class I user $=m R_{0}$, where $R_{0}$ is the basic rate, which is equal to the transmission rate of a class II user. We assume that the number of users in class I is $N_{1}$ and that in class II is $N_{2}$, with $N_{2} \geq N_{1}$. Indeed, normally the users requesting lower rate are greater than those requesting higher rate. Of course $N_{1}+N_{2}=N$. Available codes are divided equally into two pools of codes. An active user (with activity $A$ ) is assigned a code from the corresponding pool (if it belongs to class II) or assigned $m$ codes from the other pool (if it belongs to class I).

In this paper we consider two different protocols: Pro1 and Pro2. In the first protocol (Pro 1), the assigned codes are removed from the pool until the transmission is completed successfully. However, for the second protocol (Pro 2), the codes are never removed from the pool; rather a code is randomly cyclic shifted around itself once selected. A positive acknowledgment is sent from the receiver when the transmission is successful then the codes are released. Otherwise, the receiver asks for retransmission and the user enters a backlogged state. After a random time delay (with average $d$ time slots), backlogged users retransmits the $m$ or 1 packet(s) according to the class they belong to.

\section{A. System Throughput}

We evaluate the system throughput following similar methods as in [5] and [6]. Multiple access interference are taken into account in our analysis; whereas, other sources of noise are neglected. Since the number of backlogged users in each class is random, the discrete Markov chain analysis is adopted in order to evaluate the steady state throughput. We are able to show that at a given time slot, the number of backlogged packets are $m \cdot n 1$ and $n$ for classes I and II, respectively. The overall system throughput is $\beta_{\text {overall }}=\beta_{I}+\beta_{I I}$, where

$$
\begin{aligned}
& \beta_{I}\left(n_{1}, n_{2}\right)=\sum_{l=0}^{N_{2}-n_{2}} \sum_{k=0}^{n_{2}} P_{t h}\left(k \mid n_{2}\right) P_{b l}\left(l \mid n_{2}\right) \\
& \times\left[\sum_{j=0}^{\left(N_{1}-n_{1}\right) \sum_{i=0}^{n_{1} m}}\left[(i+j) \wedge C_{I}\right] P_{s}((i+j+k+l) \wedge C)\right. \\
& \left.\times P_{t h}\left(j \mid n_{1}\right) P_{b l}\left(i \mid n_{1}\right)\right]
\end{aligned}
$$

and $\beta_{I I}\left(n_{1}, n_{2}\right)$ is calculated in the same manner.

$$
\begin{aligned}
& \beta_{I I}\left(n_{1}, n_{2}\right)=\sum_{j=0}^{\left(N_{1}-n_{1}\right)} \sum_{i=0}^{m} P_{t h}\left(k \mid n_{1}\right) P_{b l}\left(l \mid n_{1}\right) \\
& \times\left[\sum_{l=0}^{N_{2}-n_{2}} \sum_{k=0}^{n_{2}}\left[(k+l) \wedge C_{I I}\right] P_{s}((i+j+k+l) \wedge C)\right. \\
& \left.\times P_{t h}\left(k \mid n_{2}\right) P_{b l}\left(l \mid n_{2}\right)\right]
\end{aligned}
$$

Here, $P_{t h}$ and $P_{b l}$ denote the probabilities of backlogged and thinking users, respectively as in [5], $P_{S}$ is the packet success probability for the correlation receiver or chip-level receiver, $C_{I}, C_{I I}$, and $C$ are the number of codes available in class I, class II, and the whole network, respectively [5], $m$ is the number of codes/user in high rate class (class I), $N_{1}$, and $N_{2}$ are the number of users in class I and class II, respectively. The packet success probability given $r$ active users is derived as in [5] for the correlation receiver:

$$
\begin{aligned}
& P_{s}(r)=\sum_{l=0}^{r-1} \sum_{m=0}^{r-1-l} \frac{(r-1) !}{l ! m !(r-1-m-l) !} \cdot p_{1}^{l} p_{w}^{m} \\
& \cdot\left(1-p_{1}-p_{w}\right)^{r-1-l-m}\left[\frac{1}{2}+\frac{1}{2^{l+m+1}} \sum_{i=0}^{w-1}\left(\begin{array}{c}
l \\
i
\end{array}\right)\right]^{K}
\end{aligned}
$$

and for the chip-level receiver:

$$
\begin{aligned}
& P_{s}(r)=\sum_{l=0}^{r-1} \sum_{m=0}^{r-1-l} \frac{(r-1) !}{l ! m !(r-1-m-l) !} \cdot p_{1}^{l} p_{w}^{m} \\
& \cdot\left(1-p_{1}-p_{w}\right)^{r-1-l-m} \cdot \sum_{l_{1}, l_{2}, \ldots, l_{w}} \frac{l !}{l_{1} ! \ldots l_{w} !} \cdot\left(\frac{1}{w}\right)^{l}\left[\frac{1}{2}+\right. \\
& \left.\frac{1}{2^{m+1}}\left(\sum_{i=1}^{w} \frac{1}{2^{l_{i}}}-\sum_{i=1}^{w-1} \sum_{j=i+1}^{w} \frac{1}{2^{l_{i}+l_{j}}}+\ldots+(-1)^{w-1} \frac{1}{2^{l}}\right)\right]^{K}
\end{aligned}
$$

where $K$ is the number of bits/packet, and $p_{1}$ and $p_{w}$ are the probabilities of one and $w$ chip interference of the 2D OCFHC/OOC respectively:

$$
\begin{aligned}
& p_{1}= \begin{cases}\frac{\left(\frac{w^{2}}{N_{o o c}}\right) t}{(C-1)} & \text { Prol } \\
\frac{\left(\frac{w^{2}}{N_{o o c}(C-1)+\frac{w(w-1)}{N o o c}}\right) t}{C} & \text { Pro2 } \\
(C-1) & \text { Prol }\end{cases} \\
& p_{w}= \begin{cases}0 & \text { Pro2 } \\
\frac{\left(\frac{1}{N_{o o c}} \cdot \frac{1}{C}\right) t}{(C-1)}\end{cases}
\end{aligned}
$$

(2) Here, $C$ is the cardinality of OCFHC/OOC as given in (1), $t=N_{\text {ooc }}-1 / w(w-1)$, and $l$ and $m$ are the number of users interfering at one and $w$ chips, respectively.

Acquiring the multi-coding technique to achieve multi-rate depends on offering each user a number of codes depending on the rate requested. This requires a large set of codes which makes the OOC [7], [8] insufficient in such case; as increasing the cardinality means increasing the code length, which is not practical for high bit rate applications where the number of time slots is limited. That is the reason behind choosing the OCFHC/OOC. Furthermore, the OCFHC/OOC correlation constraints guarantee that the codes interfere by only one chip at most [4]. 


\section{B. Average Packet Delay}

The average packet delay $D$, represents the average number of time slots a packet will be received successfully after them. $D$ is derived as:

$$
D_{i}=1+\frac{1}{\beta_{i}} \sum_{n=0}^{N_{i}} n \Pi_{n}
$$

where $i$ is the class number, $N_{i}$ is the number of users in class number $i, i \in\{1,2, \ldots\}$ and $\Pi_{n}$ is the stationary probability distribution, $n \in\left\{0,1, \ldots, N_{i}\right\}$.It can be obtained from the following equations:

$$
\sum_{n=0}^{N_{i}} \pi_{n}=1 \quad\left(\forall m \in\left\{0,1, \ldots, N_{i}\right\}\right), \sum_{n=0}^{N_{i}} \pi_{n} P_{n m}=\pi_{m}
$$

where $P_{n m}$ is the transition probability of backlogged users from state $n$ to state $m$ as in [5].

\section{IV.NUMERICAL RESULTS}

\section{A. Two Classes Network (Single Rate, Multi Rate)}

Our analysis is evaluated for a network of 30 users, $N_{1}=10$ users in class I and $N_{2}=20$ users in class II. We use a $2 \mathrm{D}$ OCFHC/OOC code with $p=2, k=2, N_{\text {OoC }}=7$, and $w=3$. Substituting in (1), we get a total cardinality of 12 codes distributed equally between the two classes $C_{I}=$ $C_{I I}=6$. The total throughputs of the network versus the activity of users for the two different protocols (Pro1 and Pro2) for both the correlation and chip-level receivers are plotted in Figs. 1, 2, respectively. It can be seen that as the rate of the users in class I increases, the throughput improves.

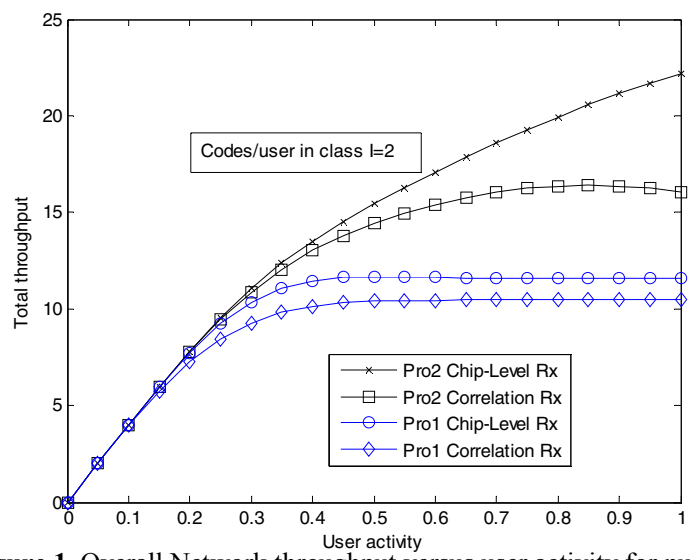

Figure 1. Overall Network throughput versus user activity for number of codes per user in class I equal 2.

For Pro1, the throughput reaches saturation because the codes available are less than the total number of users which means that a new user is allowed to access the network only when a code is released. For that reason the throughput will saturate after the available number of codes are assigned. While for Pro2 the total throughput increases until it reaches a maximum value and then decreases with increasing the users activity due to increase the interference probability. It can be seen that when using the correlation receivers, the total throughput of the two protocols are matched to each other. It appears that Pro1 is appropriate for correlation receivers. On the other hand, when using chip-level receiver, the total throughput of Pro2 is better than Pro1 for almost all activities. Moreover, the rate of the decay of the total throughput for Pro2 as the activity of the users increase for chip-level receiver is slower than its correspondent for correlation receiver due to the powerful capability in attacking multiple access interference the chiplevel receivers have.

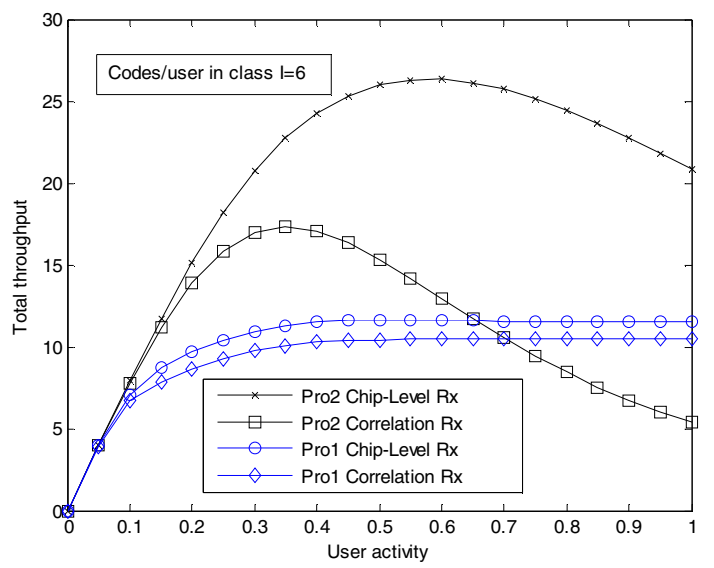

Figure 2. Overall Network throughput versus user activity for number of codes per user in class I equal 6.

The packet delay is plotted in Fig. 3. Figure 3a represents the maximum delay $D_{\max }=\max \left\{D_{I} ; D_{I I}\right\}$, where $D_{I}$ and $D_{I I}$ are the average packet delays for classes I and II respectively [5]. From the figure we can notice that for different values of $m$ the delay is the same; which declares that the higher delay is due to the low rate class. Furthermore, the average delay of the network is compared to the single class network in Fig. 3b. The results show the significant improvement in the packet delay adopting the multi-rate network. Our comparison was calculated for Pro 2 and correlation receiver.

\section{B. General Number of Classes}

To generalize our case study, users of different rates are allowed to access the network. They are divided into classes; each operating on a different rate (different values for $m$ ). In our calculations we consider a three and four class networks and compare the results with the single rate network [5]. We choose the values of $m$ of the three class network to be $\{3,2,1\}$, respectively for each class. They are $\{4,3,2,1\}$ for the four class network. We distribute the users randomly among the classes and consider the large number of user in the low rate class as the usually demanded. The total throughput is plotted in Fig. 4 using chip-level receiver. Figures $4 \mathrm{a}$ and $4 \mathrm{~b}$ are for Prol and Pro 2, respectively. It is clear that using Pro 2 makes a noticeable improvement in the network performance. An improvement in the throughput is noticed as the number of classes increased, allowing more rates to access the network. This proves that our proposed network not only supports different-rate users but even improves the total throughput. Finally, Fig. 5 illustrates the average delay of the network. It 
can be seen that as the number of classes increase the delay decreases.

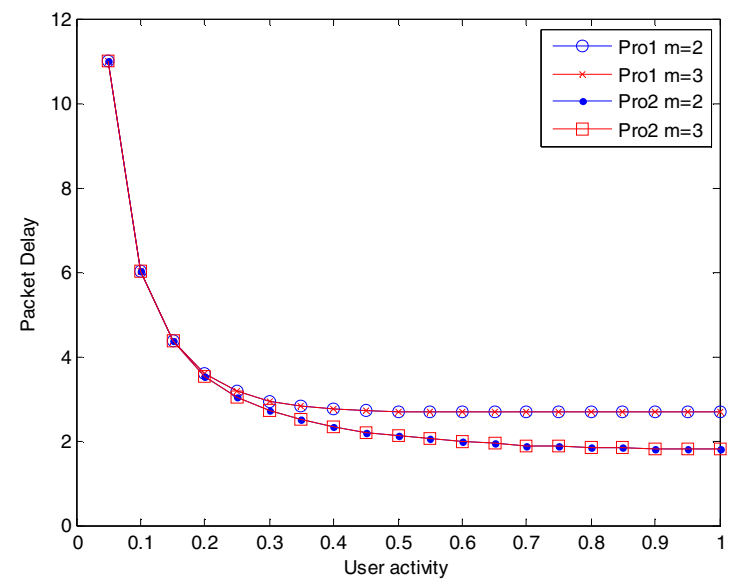

(a) Maximum packet delay

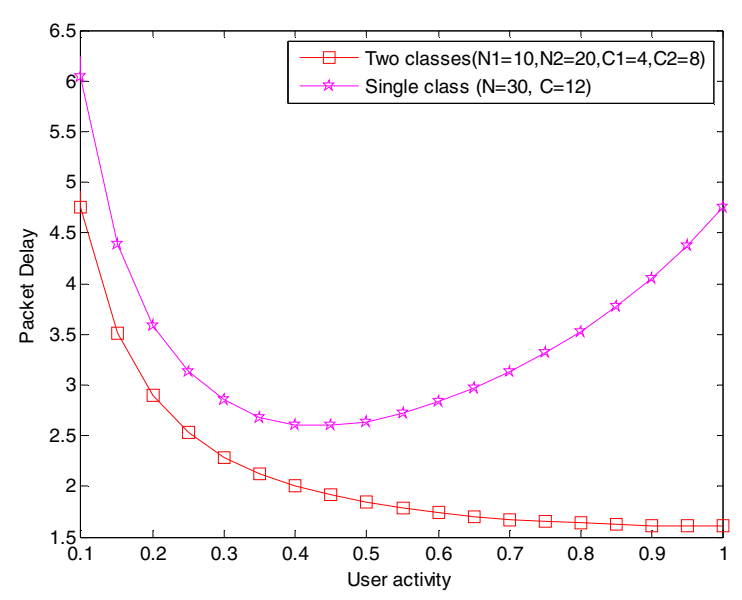

(b) Average packet delay applying Pro 2

Figure 3. Packet delay using Correlation receiver

\section{CONCLUSION}

Two random-access protocols have been proposed for multirate OCDMA networks. Multicoding technique is utilized to support different-classes (or different rates) in the network. System throughput and average packet delay have been derived. Our results reveal that the multi-rate network performs better than a single-rate network under the developed protocol for two cases as shown in the numerical results. Also as number of classes increases, both the system throughput and delay would improve.

\section{REFERENCES}

[1] T. R. Raddo, A. L. Sanches, J. V. dos Reis, and B.-H. Borges, "A new approach for evaluating the BER of a multirate, multiclass OFFH CDMA system," Communications Letters, IEEE, vol. 16, no. 2, pp. 259-261, 2012.

[2] S. Zou, M. M. Karbassian, and H. Ghafouri-Shiraz, "Extended 2D Codes Supporting Multirate and QoS in Optical CDMA Networks With Poisson and Binomial MAI Models," Journal of Optical Communications and Networking, vol. 5, no. 5, pp. 524-531, 2013.

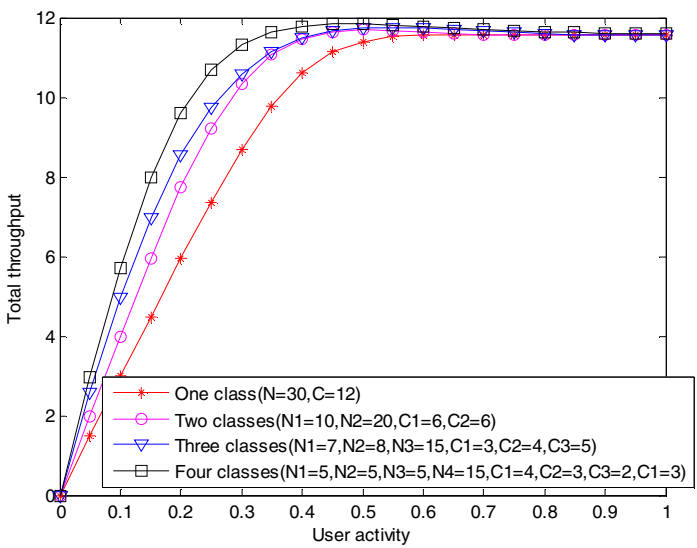

(a) For Pro1.

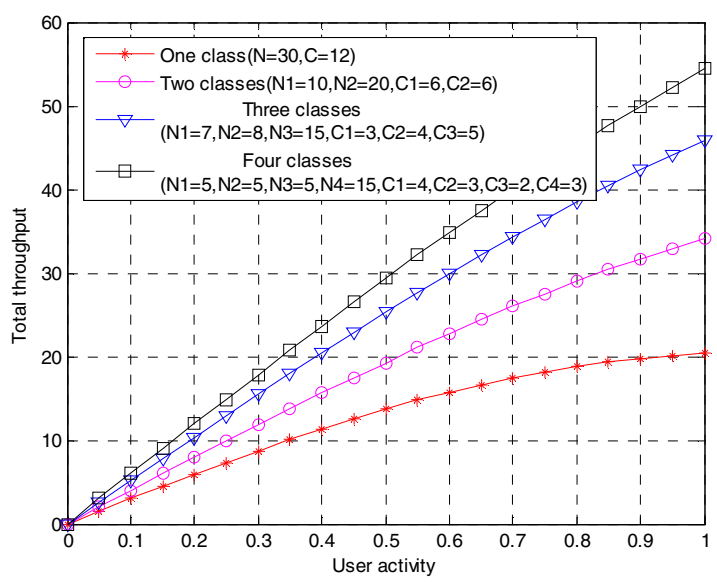

(b) For Pro2.

Figure 4. Total throughput vs user activity for Chip-Level receiver (General Classes Networks).

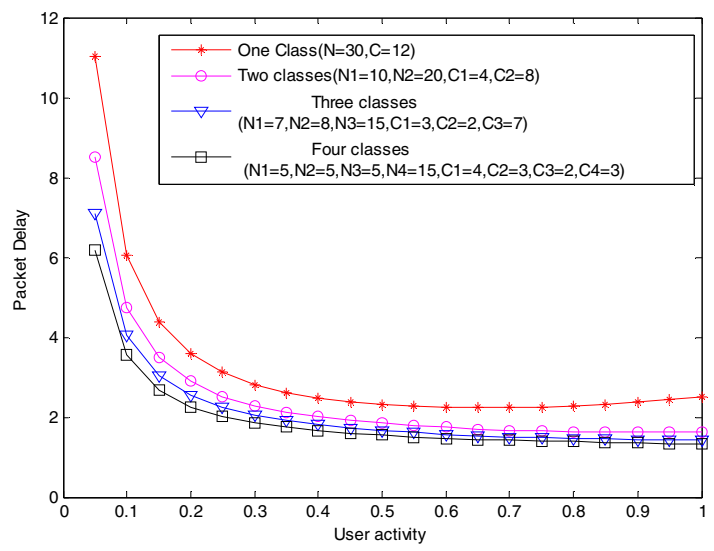

Figure 5. Average Packet Delay vs. user activity for Pro2 Correlation Receiver (General Classes Network)

[3] T. Ohtsuki, "Performance of multicode direct-detection optical CDMA systems," in Global Telecommunications Conference, 1998. GLOBECOM 1998. The Bridge to Global Integration. IEEE, vol. 6. IEEE, 1998, pp. 3227-3232.

[4] S. Shurong, H. Yin, Z. Wang, and A. Xu, "A new family of 2-D optical orthogonal codes and analysis of its performance in optical CDMA 
access networks," Journal of lightwave technology, vol. 24, no. 4, p. 1646, 2006.

[5] H. M. Shalaby, "Optical CDMA random access protocols with and without pretransmission coordination," Journal of lightwave technology, vol. 21, no. 11, p. 2455, 2003.

[6] A. Sandouk, H. Okada, T. Yamazato, M. Kayayama, and A. Ogawa, "Throughput improvement of a dual-class multi-code CDMA ALOHA system with modified channel load sensing protocol," in Communications, 1999. ICC99. 1999 IEEE International Conference on, vol. 2. IEEE, 1999, pp. 1079-1083.

[7] F. R. K. Chung, J. A. Salehi, and V. K. Wei, "Optical orthogonal codes: Design, analysis and applications," Information Theory, IEEE Transactions on, vol. 35, no. 3, pp. 595-604, 1989.

[8] J. A. Salehi, "Code division multiple-access techniques in optical fiber networks. i. fundamental principles," Communications, IEEE Transactions on, vol. 37, no. 8, pp. 824-833, 1989.

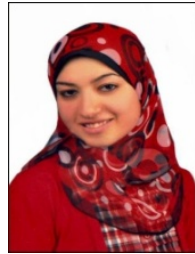

Amira M. Shata was born in Alexandria, Egypt, in 1988. He received the B.S. degrees from Alexandria University, Alexandria, Egypt. He is currently pursuing the M.S. degree at the Electrical Engineering, communication department, Alexandria University, Alexandria, Egypt. In 2013, she got a job a researcher assistant at city of

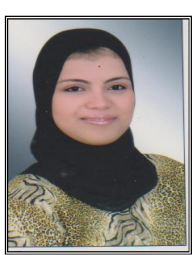

Shimaa A. Mohamed was born in Alexandria, Egypt, in 1988. He received the B.S. degrees from Alexandria University, Alexandria, Egypt. He is currently pursuing the M.S. degree at the Electrical Engineering, communication department, Alexandria University, Alexandria, Egypt. In 2012, she got a job a researcher assistant at city of Scientific Research and Technological Applications, Informatics Institute.

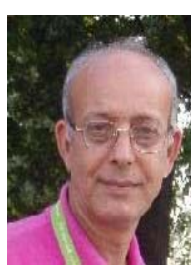

Ahmed Abdel Nabi is an Associated Professor at City of Scientific Research and Technological Applications, Informatics Institute, Head of Network and Distributed Systems Department, Alexandria Egypt.

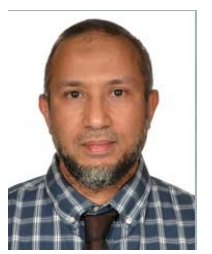

Hossam M. H. Shalaby (S'83-M'91-SM'99) was born in Giza, Egypt, in 1961. He received the B.S. and M.S degrees from Alexandria University, Alexandria, Egypt, in 1983 and 1986, respectively, and the Ph.D. degree from the University of Maryland at College Park in 1991, all in electrical engineering. In 1991, he joined the Electrical Engineering Department, Alexandria University, and was promoted to Professor in 2001. Currently he is on leave from Alexandria University, where he is the chair of the Department of Electronics and Communications Engineering, School of Electronics, Communications, and Computer Engineering, Egypt-Japan University of Science and Technology (E-JUST), New Borg El-Arab City, Alexandria, Egypt. From December 2000 to 2004, he was an Adjunct Professor with the Faculty of Sciences and Engineering, Department of Electrical and Information Engineering, Laval University, Quebec, QC, Canada. From September 1996 to February 2001, he was on leave from Alexandria University. From September 1996 to January 1998, he was with the Electrical and Computer Engineering Department, International Islamic University Malaysia, and from February 1998 to February 2001, he was with the School of Electrical and Electronic Engineering, Nanyang Technological University, Singapore. 